\title{
Intravenous fluid use in the acutely unwell adult medical inpatient: improving practice through a clinical audit process
}

\author{
${ }^{1} \mathrm{GE}$ Walker, ${ }^{2} \mathrm{E}$ Stewart-Parker, ${ }^{2} \mathrm{~S}$ Chinthapalli, ${ }^{3} \mathrm{M}$ Ostermann, ${ }^{4} \mathrm{PI}$ Dargan, ${ }^{5} \mathrm{DM}$ Wood \\ ${ }^{1}$ ST2 Trainee, ACCS Training Programme; ${ }^{2}$ Foundation Trainee; ${ }^{3}$ Consultant in Nephrology \& Intensive Care, Guy's and St Thomas' NHS \\ Foundation Trust and King's Health Partners, London, UK; ${ }^{4}$ Consultant Physician and Clinical Toxicologist, Guy's and St Thomas' NHS \\ Foundation Trust and King's Health Partners, London, UK and Reader, King's College London, UK; ${ }^{5}$ Consultant Physician and Clinical \\ Toxicologist, Guy's and St Thomas' NHS Foundation Trust and King's Health Partners, London, UK and Senior Lecturer, King's College \\ London, UK
}

\section{ABSTRACT}

Background: Our Trust developed a clinical guideline to improve the prescribing and use of intravenous (IV) fluids based on the British consensus guidelines on IV fluid therapy for adult surgical patients. We audited the effect of targeted interventions to improve performance against this guideline.

Method: There were 53 IV fluid prescription charts in the pre-intervention audit and 48 in the post-intervention audit. Data was collected on the seven local practice standards ('local gold standards') in the clinical guideline; compliance with all of them was necessary to meet the IV fluid prescribing bundle of care.

Results: The proportion of prescriptions which met the IV fluid prescribing bundle of care increased $(3.8 \%$ to $22.9 \%[p=0.004])$ and the legibility of the IV fluid prescription increased $(28.3 \%$ to $56.3 \%[p=0.004])$.

Conclusion: We have shown that the process of prescribing, administering and monitoring IV fluid use can be significantly improved through a range of targeted multi-disciplinary interventions.

KEYWORDS Intravenous fluids, clinical audit, education, prescribing, guidelines

DECLARATION OF INTERESTS No conflicts of interest declared.
Correspondence to DM Wood Clinical Toxicology

3rd Floor, Block C, South Wing St Thomas' Hospital

London SEI 7EH, UK

tel. $+44(0) 207$ I 885848

e-mail David.Wood@gstt.nhs.uk

\section{INTRODUCTION}

A significant proportion of patients admitted to hospital require the administration of intravenous (IV) fluids as part of their medical management. These may be required for fluid replacement (e.g. due to dehydration or gastrointestinal haemorrhage), management of an underlying medical condition (e.g. diabetic ketoacidosis), for administration of medications (e.g. IV antibiotics) or maintenance hydration in those who are receiving nil by mouth. In seriously ill patients, fluid resuscitation is the second most common intervention (after oxygen therapy). Sometimes the choice of fluid is specific to the indication, for example $0.9 \%$ sodium chloride solution for patients with diabetic ketoacidosis during the initial management, or blood replacement for upper gastrointestinal haemorrhage. However, there is often conflicting advice on the appropriate fluids for maintenance therapy. In severely ill patients there is increasing recognition that the choice of fluid may affect outcome; this has led to the publication of fluid guidelines and recommendations. ${ }^{1-4}$ There is also the need for regular clinical assessment, to ensure that not only is it appropriate to continue with IV fluids, but also that the patient is not being overloaded or becoming volume depleted, leading to pulmonary oedema or pre-renal acute kidney injury, respectively. With the exception of $5 \%$ dextrose solutions, almost all intravenous solutions contain sodium and chloride - some in near physiological concentrations of 140 millimoles per litre $(\mathrm{mmol} / \mathrm{L})$ of sodium and $95 \mathrm{mmol} / \mathrm{L}$ of chloride, others in supranormal amounts e.g. $154 \mathrm{mmol} / \mathrm{L}$ of sodium and $154 \mathrm{mmol} / \mathrm{L}$ of chloride in $0.9 \%$ sodium chloride solution (it is well recognised that this is often incorrectly referred to as normal saline, whereas in reality it is neither 'normal' nor 'physiological')'. Studies in healthy volunteers and patients undergoing major surgery have demonstrated significant hyperchloraemia following administration of $0.9 \%$ sodium chloride, leading to hyperchloraemic metabolic acidosis, an increased incidence of postoperative nausea and vomiting and a temporary reduction in the glomerular filtration rate..$^{6-8}$ It is estimated that in healthy volunteers it can take up to two days to excrete a rapid infusion of two litres of $0.9 \%$ 
sodium chloride solution; ${ }^{9}$ in hospital inpatients administered IV fluids for prolonged periods of time, this can increase the risk of fluid overload and associated complications. Studies in surgical patients have shown that the use of $0.9 \%$ sodium chloride solution compared to balanced fluid solutions is associated with an increased in-hospital mortality (5.6\% compared to $2.9 \%$, p $<0.001) .{ }^{10}$ Additionally, the use of $0.9 \%$ sodium chloride was more likely to be associated with one or more major complication(s) compared to balanced fluid solutions (33.7\% compared to $27 \%, \mathrm{p}<0.0001) .{ }^{10}$ Consequently, physiologic (balanced) fluid solutions like Ringer's lactate or Hartmann's fluid are recommended for most clinical situations; in particular the recent Cochrane review on fluid resuscitation in critically ill patients recommended that crystalloid solutions should be used in preference to colloid solutions and did not support the use of colloids outside of clinical trials. ${ }^{1-4}$

In hospital patients, there is increasing evidence of significant deficiencies in the quality of the process of prescribing, dispensing and administering of medications. ${ }^{11-15}$ One inaccuracy may not lead to significant patient harm, but when multiple errors occur together, there can be a significant risk of morbidity and potentially death (for example the mis-prescribing and administration of co-amoxiclav in a patient with a history of penicillin allergy). Thus measures are often undertaken to increase awareness of the dangers of inappropriate prescribing. However, less attention is given to the prescribing of IV fluids, which may often be done by the more junior medical staff. Previous studies of junior medical staff have shown that pre-registration house officers (equivalent to foundation year one [FYI] doctors) were responsible for $89 \%$ of IV fluid prescribing. ${ }^{16}$

In order to improve the process of IV fluid prescribing in hospital inpatients, our Trust, using a multi-disciplinary approach, developed and implemented a clinical guideline entitled Fluid Replacement Guideline for Adult Patients on Medical and Surgical Wards. This also incorporated advice from the British Consensus Guidelines on Intravenous Fluid Therapy for Adult Surgical Patients (GIFTASUP);' it did not incorporate all 28 GIFTASUP standards but attempted to incorporate those standards which we felt could lead to improved patient care and reduced morbidity from IV fluid use if appropriately followed. Our clinical guideline had seven key local practice standards ('local gold standards'):

I. All fluids prescribed should have a clear indication documented.

2. Compound sodium lactate, or Hartmann's solution, should be the fluid of choice in most circumstances unless there is a specific reason for an alternative.

3. All patients on IV fluids should have their fluid balance assessed and documented by the medical team daily.
4. All patients should have their fluid balance documented on the input/output charts daily.

5. If a patient is found to require IV fluid management, the rate of replacement should be based on the patient's weight.

6. A $0.9 \%$ sodium chloride solution should not be prescribed as N/Saline, normal saline or $0.9 \%$ normal saline solution.

7. If a fluid challenge is required, for example in sepsis, Hartmann's should be selected as the first-line fluid.

These local practice standards were designed to improve the process of prescribing IV fluids by ensuring that there was an indication for the use of IV fluids, that the appropriate IV fluid was used, that there was appropriate assessment of fluid balance and the ongoing need for IV therapy and that the prescribing process was accurate. We undertook a clinical audit to determine the preguideline adherence to the local practice standards; we then implemented a number of targeted interventions in an attempt to address areas of deficiencies in practice as identified in the initial analysis and then re-audited after implementation. We report here the outcomes of this clinical audit process.

\section{METHODS}

\section{Audit assessment process}

Patients admitted to our two acute admitting wards during October and November 2010 (for the baseline audit) and June and July $20 \mathrm{II}$ (for the follow-up audit) were considered for inclusion. Data were collected twice a week during the audit period; patients were included if they had been prescribed and/or received IV fluids in the preceding 24 hours. The medical and nursing records and documentation, as well as the inpatient prescription chart were reviewed to extract the following information:

I. Documentation of the indication for IV fluids.

2. Use of Hartmann's solution unless there was a specific reason for alternative fluid choice.

3. Medical assessment of fluid balance status.

4. Completion of input/output fluid charts.

5. Weight documented in relation to the admission.

6. Legibility of the prescription for IV fluids.

7. The appropriate use of Hartmann's solution for fluid challenge.

For each patient, our target for the IV fluid prescribing bundle of care was full compliance with all the practice standards of the clinical guideline; if any one standard was not achieved a patient was deemed to have failed to meet the target. 
Following analysis of the baseline audit data and internal review, a number of interventions were delivered to improve the areas of deficiencies in comparison with the local practice standards. These interventions were targeted at all professional groups involved in the process of prescribing and administering IV fluid. The interventions included:

I. The broad findings of the baseline audit were presented at acute medicine directorate clinical effectiveness/audit meetings; these included representatives from emergency medicine, acute medicine and general internal medicine and were multi-professional, involving doctors, nurses and pharmacists.

2. The key areas of deficiency in the use of IV fluids were communicated to all Trust staff, through the use of the Trust computer network. In our Trust, all computers connected to the Trust intranet server can display the same background wallpaper and this is used to highlight key issues/messages to staff. A sample screenshot of the computer wallpaper is shown in Figure I. We used this method intermittently during the intervention phase to highlight the key messages from the initial audit and also to provide a link to the IV fluid guideline.

3. The inclusion of a prompt on the general medicine admission proforma to review IV fluid prescribing and fluid balance assessment.

4. Targeted teaching of foundation year I and 2 and specialist trainee year $I$ and 2 junior medical staff (responsible for the majority of IV fluids prescription) to highlight the clinical guideline and the reasons for the choice of Hartmann's solution as the preferred IV fluid for maintenance therapy.

5. The inclusion of the IV guidelines in the handbook given to junior medical staff at the commencement of their placement at the Trust.

\begin{tabular}{|c|}
\hline Guy's and St Thomas' NHS \\
Prescribing IV fluids to adult patients? \\
Have you read the Trust's fluid replacement guidelines? \\
Think: \\
1. What is the indication for fluids? \\
2. Has fluid balance been assessed today? \\
3. Are you using the correct fluids? \\
Read the guidelines at http://gti/adultivfluid
\end{tabular}

FIGURE I Trust computer wallpaper used for generalised dissemination of the key areas relating to deficiencies in the appropriate use of IV fluids (Guy's and St Thomas' NHS Foundation Trust).

6. Communication of the importance of complete documentation of fluid balance charts and recording of weight as one of the local 'nursing big four' items to be discussed at all nursing staff handovers.

\section{Data analysis}

Collected audit data was entered into an Excel spreadsheet; results are presented as percentages and comparison between pre- and post-intervention data collection variables was by $\mathrm{Chi}$-squared analysis.

\section{RESULTS}

\section{Study population}

The baseline audit phase included 53 patients and 48 were in the follow-up group after the delivery of the interventions described above.

TABLE I Proportion of IV fluid prescriptions complying with each practice standard variable, pre- and post-interventions

\begin{tabular}{|l|l|c|c|c|}
\hline Standard & \multicolumn{1}{|c|}{ Description } & $\begin{array}{c}\text { Pre-intervention } \\
\text { compliance }\end{array}$ & $\begin{array}{c}\text { Post-intervention } \\
\text { compliance }\end{array}$ & P value \\
\hline 1 & $\begin{array}{l}\text { Indication for IV fluids documented in } \\
\text { medical notes }\end{array}$ & $81.1 \%$ & $87.5 \%$ & 0.38 \\
\hline 2 & $\begin{array}{l}\text { Use of Hartmann's solution unless other } \\
\text { fluid indication }\end{array}$ & $39.6 \%$ & $60.4 \%$ & 0.037 \\
\hline 3 & Medical assessment of fluid balance & $62.3 \%$ & $70.8 \%$ & 0.36 \\
\hline 4 & $\begin{array}{l}\text { Completion of fluid balance charts by } \\
\text { nursing staff }\end{array}$ & $62.3 \%$ & $70.8 \%$ & 0.36 \\
\hline 5 & Weight recorded by nursing staff & $28.3 \%$ & $60.4 \%$ & 0.001 \\
\hline 6 & $\begin{array}{l}\text { Correct prescribing of 0.9\% sodium chloride } \\
\text { solution }\end{array}$ & $12.2 \%$ & $21.7 \%$ & 0.31 \\
\hline 7 & $\begin{array}{l}\text { Hartmann's solution used for IV fluid } \\
\text { challenge }\end{array}$ & $28.6 \%$ & $100 \%$ & 0.038 \\
\hline
\end{tabular}




\section{Impact of interventions to improve practice}

Compliance with the individual local practice standards of the clinical guideline during the baseline and the postintervention audit phases are shown in Table I. There was a significant improvement in the use of Hartmann's solution for IV maintenance therapy and also for fluid challenges, unless there was a specific indication for an alternative IV fluid. The recording of weight by nursing staff also improved. Documentation of the indication for IV fluid therapy also improved but since the baseline compliance with this standard was better than for the others, this improvement was not of significance.

Overall, the proportion of patients who met the IV fluid prescribing bundle of care target increased from 3.8\% during the baseline audit period to $22.9 \%$ after the interventions $(p=0.004)$ as did the legibility of the IV fluid prescription (28.3\% pre-intervention compared to $56.3 \%$ post-intervention, $p=0.004$ ). Additionally, assessment of fluid balance status by medical staff was more likely to be undertaken when the nurses had completed the fluid balance charts compared to when these were not completed $(73 \%$ vs $53 \%, p=0.04)$.

\section{DISCUSSION}

This clinical audit has shown that prescribing, administering and monitoring of IV fluid use can be significantly improved through a range of targeted multidisciplinary interventions. However, we feel that the implementation of the Trust clinical guideline alone is insufficient to completely explain this improvement, given the poor baseline compliance with the local practice standards for IV fluid. It should be noted that even with targeted interventions, there were still areas of poor performance and the overall IV fluid guideline bundle of care compliance rate was only $22.9 \%$.

There is an increasing use of guidelines throughout areas of medical practice, with the expectation that they will lead to improvement and standardisation. There is however the possibility that healthcare professionals may become saturated with the number of guidelines that must be followed on a daily basis. Additionally, with increasing numbers, there is the possibility that individuals are unaware of specific guidelines that they should follow. This can be a barrier to guideline adherence. ${ }^{17,18}$ Healthcare professionals with training and/or previous work outside our Trust may have learned different practices; there can be a reluctance to change their own practice, particularly if they feel that the clinical guidelines are not in line with their own experiences or guidelines used previously (they are often specific to individual Trusts)..$^{17,18}$ We tried to address these potential barriers related to the application of the IV fluid guideline during the implementation phase, using a number of targeted interventions. Our aim was to increase overall awareness of the guideline and to explain the scientific and medical basis for its development so that the process was seen as robust and valid.

National guidelines, often introduced by the National Institute for Health and Clinical Excellence (NICE) or speciality societies, tend to have better, longer term application due to a more robust implementation process, often supported by compulsory national audits. ${ }^{19} \mathrm{New}$ local clinical guidelines therefore need to both create and maintain awareness of the guideline and facilitate compliance. We planned longer-term interventions, hoping to achieve and maintain compliance outside of this audit. First we introduced a check box on the general medicine admission post-take ward proforma. This highlights that at this stage the consultant in particular should review the appropriateness for continuing IV fluid administration and assess the fluid balance status and choice of IV fluids. Additionally, information on the guideline and guidance on IV fluid management was noted in the handbook given to new junior medical staff, providing ongoing dissemination of the information.

When designing interventions in a clinical audit process it is important to consider whether these should be targeted to individual healthcare groups or across the multi-disciplinary team. It is possible that improved standards in one healthcare group can lead to improvement in others. In our audit process we noticed that accurate completion of fluid balance charts by nursing staff led to a greater assessment and documentation of fluid balance status by medical staff compared to patients where the charts had not been completed. This clearly demonstrates that careful consideration is needed to ensure that interventions in clinical audit are appropriately targeted across the multidisciplinary team.

In this study we introduced a number of interventions targeted at different healthcare professional groups and across different stages of the IV fluid pathway. Therefore, while we demonstrated that there was an overall improvement in the use of IV fluids, we were not able to determine which interventions were most responsible. Studies looking at the targeted introduction of single interventions in a stepwise approach to determine the optimum combination of interventions to improve clinical practice should be undertaken. It is possible that this approach will demonstrate that varied approaches are needed for different areas of clinical practice and healthcare professional groups. 


\section{REFERENCES}

I Powell-Tuck J, Gosling P, Lobo DN et al. British consensus guidelines on intravenous fluid therapy for adult surgical patients (GIFTASUP) [Internet]. 20I I [cited 2012 March 26]. Available from: http://www. bapen.org.uk/pdfs/bapen_pubs/giftasup.pdf

2 Leach R. Fluid management on hospital medical wards. Clin Med 2010; 10:611-5.

3 Reinhart K, Perner A, Sprung CL et al. Consensus statement of the ESICM task force on colloid volume therapy in critically ill patients. Intensive Care Med 2012; 38:368-83. http://dx.doi.org//0.1007/ s00I34-0I2-2472-9

4 Perel P, Roberts I. Colloids versus crystalloids for fluid resuscitation in critically ill patients. Cochrane Database Syst Rev 2012;6:CD000567.

5 Awad S, Allison SP, Lobo DN. The history of $0.9 \%$ saline. Clin Nutr 2008; 27:179-88. http://dx.doi.org/I0.1016/j.clnu.2008.01.008

6 Williams EL, Hildebrand KL, McCormick SA et al. The effect of intravenous lactated Ringer's solution versus $0.9 \%$ sodium chloride solution on serum osmolality in human volunteers. Anesth Analg 1999; 88:999-1003.

7 Wilkes $\mathrm{N}$, Woolf R, Mutch $M$ et al. The effects of balanced versus saline-based hetastarch and crystalloid solutions on acid-base and electrolyte status and gastric mucosal perfusion in elderly surgical patients. Anesth Analg 200I; 93:8II-6. http://dx.doi. org/10.1097/00000539-200II0000-00003

8 Yunos NM, Bellomo R, Story D et al. Bench-to-bedside review: chloride in critical illness. Crit Care 2010; 14:226. http://dx.doi. org/I0.II86/cc9052

9 Drummer C, Gerzer R, Heer $M$ et al. Effects of an acute saline infusion on fluid and electrolyte metabolism in humans. Am J Physiol 1992; 262:F744-54.

I0 Shaw AD, Bagshaw SM, Goldstein SL et al. Major complications, mortality, and resource utilization after open abdominal surgery: 0.9\% saline compared to Plasma-Lyte. Ann Surg 20I2; 255:82I-9. http://dx.doi.org/I0.1097/SLA.0b0 I 3e31825074f5
II Baker E, Roberts AP, Wilde K et al. Development of a core drug list towards improving prescribing education and reducing errors in the UK. Br J Clin Pharmacol 20II; 7I:190-8. http://dx.doi. org/I0.IIIII/j.I365-2I25.20I0.03823.x

12 Cahir C, Fahey T, Teeling $M$ et al. Potentially inappropriate prescribing and cost outcomes for older people: a national population study. Br J Clin Pharmacol 20 10; 69:543-52. http://dx.doi. org/I0.IIIII/j.I365-2I25.20I0.03628.x

13 Members of EMERGE, Erice Medication Errors Research Group, Agrawal A,Aronson JK, Britten $\mathrm{N}$ et al. Medication errors: problems and recommendations from a consensus meeting. $\mathrm{Br}$ J Clin Pharmacol 2009; 67:592-8. http://dx.doi.org/I0. I I I //j. I365-2I25.2009.034I4.x

14 Berdot S, Sabatier B, Gillaizeau F et al. Evaluation of drug administration errors in a teaching hospital. BMC Health Serv Res 2012; 12:60. http://dx.doi.org/I0.I I86//472-6963-12-60

15 Dean B, Schachter M,Vincent $C$ et al. Causes of prescribing errors in hospital inpatients: a prospective study. The Lancet 2002; 359:1373-8. http://dx.doi.org//0.1016/S0|40-6736(02)08350-2

16 Lobo DN, Dube MG, Neal KR et al. Problems with solutions: drowning in the brine of an inadequate knowledge base. Clin Nutr 200 I; 20:I 25-30. http://dx.doi.org/I0.I054/clnu.2000.0I54

17 Cabana MD, Rand CS, Powe NR et al.Why don't physicians follow clinical practice guidelines? A framework for improvement. JAMA 1999; 282: I458-65. http://dx.doi.org//0.100I/jama.282.I5.I458

18 Francke AL, Smit MC, de Veer AJ. Factors influencing the implementation of clinical guidelines for health care professionals: a systematic meta-review. BMC Med Inform Decis Mak 2008; 8:38.

19 Johnston KN, Young M, Grimmer-Somers KA et al. Why are some evidence-based care recommendations in chronic obstructive pulmonary disease better implemented than others? Perspectives of medical practitioners. Int J Chron Obstruct Pulmon Dis 201 I; 6:659-67. http://dx.doi.org/I0.2 I47/COPD.S2658I

\section{UK CONSENSUS CONFERENCE MANAGEMENT OF ACUTE KIDNEY INJURY: THE ROLE OF FLUIDS, E-ALERTS AND BIOMARKERS Friday 16 and Saturday 17 November 2012 At the Royal College of Physicians of Edinburgh}

This two-day UK Consensus Conference on Acute Kidney Injury has been convened by the Royal College of Physicians of Edinburgh. The multidisciplinary panel, co-chaired by Professor Sir lan Gilmore and Professor John Feehally, will develop a Consensus Statement on the role of fluid therapy, e-alerts and biomarkers in AKI from the written and oral presentations, submitted abstracts and conference discussion and debate.

Additional education sessions on the patient journey and challenges in managing AKI will also be offered on the second day of the conference.

Details are online at www.rcpe.ac.uk

For more information, contact:

Margaret Farquhar, Consensus Conference Coordinator, RCPE, 9 Queen Street, Edinburgh EH2 IJQ Tel: +44 (0) I3। 2473636 E-mail: m.farquhar@rcpe.ac.uk

Kidney Care 